\title{
The crystal structure of LidA, a translocated substrate of the Legionella pneumophila type IV secretion system
}

\begin{abstract}
Dear Editor,
Legionella pneumophila, a gram-negative bacterium, is the causative agent of Legionnaires' pneumonia by infecting human lung macrophages (Muder et al., 1986). Upon uptake into macrophages, the bacteria are internalized to escape the endocytic pathway and lysosomal network, and establish the Legionellacontaining vacuole (LCV) as an intracelIular replicative organelle (Roy et al., 1998). The LCV hijacks and recruits the early secretory ER-derived vesicles and transforms them into ER-like vacuole. Within the specialized ER-like vacuole, $L$. pneumophila replicates to a high density and subsequently kills the host cell by lysing the host cell membrane. The released bacteria can infect neighboring cells to initiate a new round of infection (Tilney et al., 2001).

The formation of the LCV requires several bacterial factors that are secreted out of the bacteria through Dot/ Icm type IV secretion systems (Ensminger and Isberg, 2009). Among these secreted effectors, LidA is an essential one that determines the formation of LCV (Conover et al., 2003; Derre and Isberg, 2005). After $L$. pneumophila is internalized by host cells, LidA immediately translocates into the host cytosol via the Dot/lcm system and associates with the cytoplasmic surface of LCV membrane by binding to Ptdlns(3)P or Ptdlns(4)P (Brombacher et al., 2009). In addition, LidA can interact and recruit GDI-free Rab1 to the LCV membrane without changing the activation state of Rab1 (Machner and Isberg, 2007; Ensminger and Isberg, 2009), and synergize with SidM, another important Dot/lcm
\end{abstract}

secreted effector, to interact with Rab1 for recruitment of early secretory vesicles to the surface of LCV (Machner and Isberg, 2006; Zhu et al., 2010). Besides, LidA can also bind Rab6 and Rab8, two small GTPases regulating Golgi-to-ER and Golgi-to-plasma membrane traffic, suggesting that LidA can subvert multiple host trafficking pathways (Huber et al., 1993; White et al., 1999; Machner and Isberg, 2006). Recently published two crystal structures of the central Rabbinding domain of LidA complexed with Rab8a or Rab1 reveal a similar Rabbinding mechanism (Schoebel et al., 2011; Cheng et al., 2012), although the structural information of the other parts of LidA remains unknown.

Here we report the crystal structure of LidA at an apo-state, including both the central Rab-binding domain and an $\mathrm{N}$-terminal region. We began our study with full-length LidA and solved the phasing problem with single-wavelength anomalous dispersion (SAD) method at Se K-edge, although a further refinement was difficult to perform due to the bad data quality of full-length protein. In the preliminary model of LidA, the $\mathrm{N}$-terminal 59 residues and $\mathrm{C}$-terminal 141 residues were missing. Accordingly, we employed a truncation of $\mathrm{LidA}_{60-594}$ to promote the diffraction quality and obtained its crystals in the same crystal form with fulllength ones. The structure of $\mathrm{LidA}_{60-594}$ can be refined to a 2.1- $\AA$ resolution with only the residues between Ser181 and Ser189 absent because of the flexibility of this loop region (see Table S1 for detailed statistics). The coordinates of final model was deposited into Protein Data Bank (PDB) with an entry code of $4 \mathrm{H} 5 \mathrm{Y}$.
The overall structure of LidA ${ }_{60-594}$, with dimension of $\sim 135 \times 65 \times 55 \AA$, exhibits a novel folding pattern, which is in the shape of a human body (Fig. 1A and $1 \mathrm{~B}$ ). One can easily tell the "head" (including ah2, $\beta \mathrm{h} 1, \beta \mathrm{h} 2$ and the linker between $\alpha 7$ and $\alpha 9$, magenta in Fig. 1), the "right arm" (ah1, red in Fig. 1), the "left arm" (including $\beta \mathrm{h} 3, \beta \mathrm{h} 4, \beta \mathrm{h} 5$ and $\alpha 13$, pink in Fig. 1), the "legs" (long coiled-coil $\alpha 7$ and $\alpha 14$, blue in Fig. 1), and the "right foot" (cyan in Fig. 1).

Our crystal structure nearly covers the whole "coiled-coil domain" (Thr191Glu600) in the central region of LidA, which forms two a-helical hairpins (ah1 and ah2) and a $80-\AA-$-long antiparallel coiled-coil structure ( $\alpha 7$ and $\alpha 14)$. Nevertheless, there are also $10 \beta$-strands in the "coiled-coil domain", assembling exactly $5 \beta$-hairpins ( $\beta$ h1- $\beta$ h5). Among them, $\beta \mathrm{h} 2, \beta \mathrm{h} 4$ and $\beta \mathrm{h} 5$ are common $\beta$-hairpins, with a 2-residue $\beta$-turn connecting two antiparallel $\beta$-strands. Different from those three $\beta$-hairpins, both $\beta \mathrm{h} 1$ and $\beta \mathrm{h} 3$ possess a long interstrand loop (13 residues), which is in the shape of a square-knot, even comprising a one-turn $3_{10}$ helix. Therefore, we term $\beta \mathrm{h} 1$ and $\beta \mathrm{h} 3$ as "square-knot" $\beta$-hairpins. The "coiled-coil domain" was shown to bind Rabs and called "Rab-binding domain" also. The N-terminal right foot region from Pro60 to Ser181 consists of six short helices ( $\alpha 1-\alpha 6)$, flanked by the Rab-binding domain with a loose helical bundle formed by $\alpha 5, \alpha 6, \alpha 7$ and a14 (see Fig. 1C for detailed secondary structure arrangement).

A DALI search for structural similarity identified two unknown but similar motifs in LidA structure. One of them is the 
A
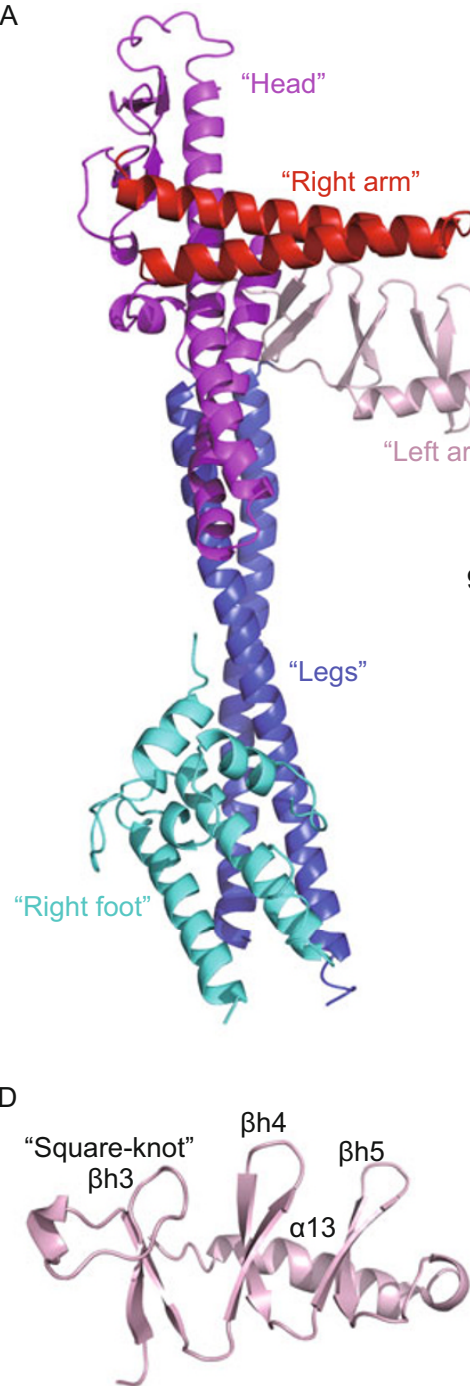

B
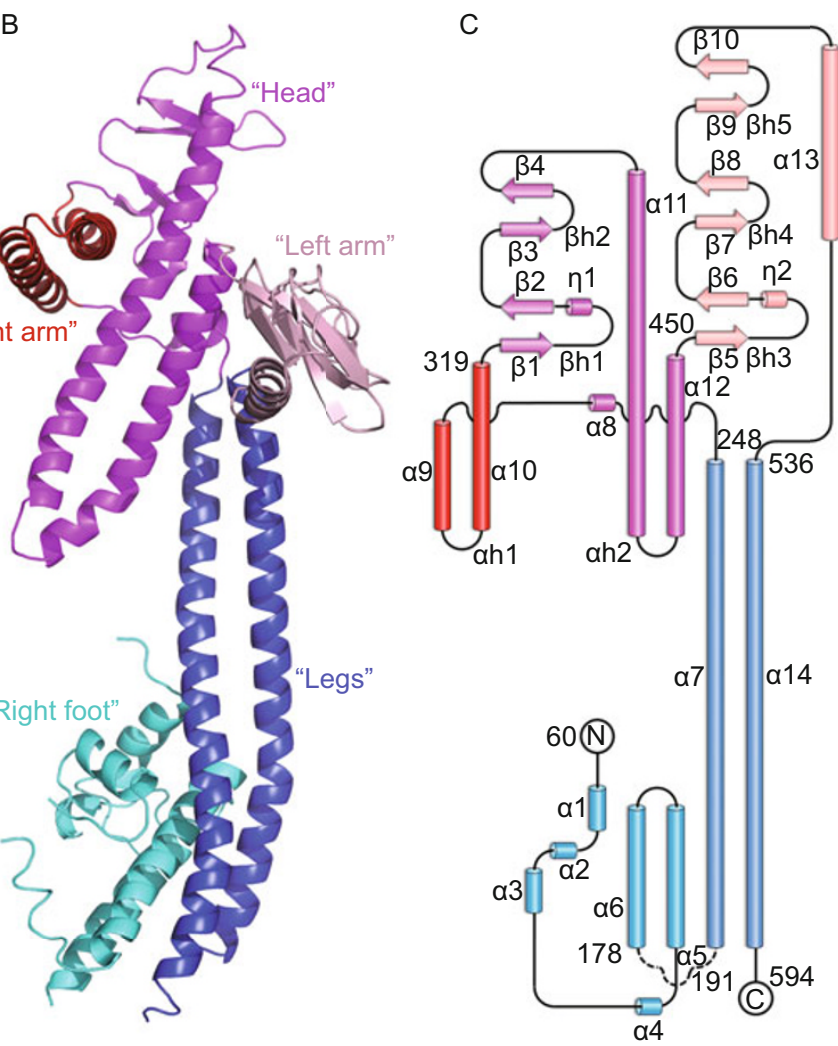

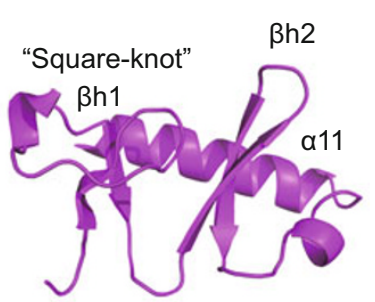

F

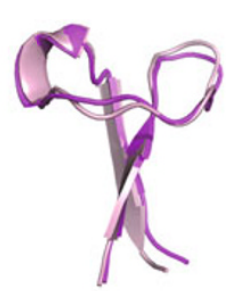

G

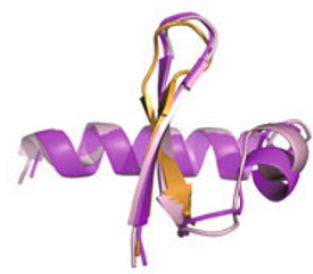

Figure 1. The overall structure of LidA $A_{60-594}$ and its TBR motifs. (A) The overall structure of LidA $A_{60-594}$ exhibits a novel folding pattern which is in the shape of a human body. The "head" is colored in magenta, as well as the "right arm" in red, the "left arm" in pink, the "legs" in blue, and the "right foot" in cyan, respectively. (B) A front view of LidA $A_{60-594}$ structure. (C) Overall topology schematic of LidA $A_{60-594}$. Secondary structures are colored in the same pattern as in (A). The molecular architecture of the full length LidA (1-735 aa) are also colored in the same pattern as in (A) and the missing part 1-60 aa and 595-735 aa are colored in white. (D) TBR motif in the "left arm" (pink).

(E) TBR motif in the "head" (magenta). (F) Superposition of the square-knot $\beta$-hairpins $\beta$ h1 and $\beta h 3$, showing high structural similarity.

(G) Superposition of $\beta \mathrm{h} 2-\alpha 11$ (magenta) and $\beta \mathrm{h} 5-\alpha 13$ (pink), together with a single $\beta$-hairpin $\beta \mathrm{h} 4$ (orange).

entire "left arm" region, comprising three twisted $\beta$-hairpins $(\beta \mathrm{h} 3, \beta \mathrm{h} 4$ and $\beta \mathrm{h} 5)$ followed by an $\alpha$-helix ( $\alpha 13$ ) on the flank of those $\beta$-hairpins (Fig. 1D). The Nterminal $\beta$ h3 is a square-knot $\beta$-hairpin, while the rest two are ordinary ones. The second unknown motif locates at the "head" part of LidA (Fig. 1E) and is structurally similar to the first one. Different from the "left arm", the "head" has only two $\beta$-hairpins, a square-knot one (Bh1) and an ordinary one (Bh2). The two tandem novel motifs show an important structural feature of LidA in addition to the coiled-coil components.

Given the obvious structural similarity between those two motifs, we tend to consider them as different cases of a novel motif and propose to term it as "twisted $\beta$-hairpin repeats" (TBR) motif. Based on the only two examples we have now, a TBR motif should contain 2 or 3 (could be more in future cases) twisted $\beta$-hairpins in parallel, flanked by a C-terminal $\alpha$-helix. The very $\mathrm{N}$-terminal $\beta$-hairpin should be a square-knot one, with a long interstrand loop possessing a one-turn $3_{10}$ helix. We believe that this square-knot $\beta$-hairpin is a characteristic feature of TBR motif. The two square-knot $\beta$-hairpins in LidA share a root mean square deviation (r.m.s.d.) of $0.70 \AA$ for 23 main-chain $\mathrm{C} \alpha$ atoms (Fig. 1F) and a high sequence similarity (Fig. S1). Actually, the "square-knot" displays an amino acid sequence pattern 
A
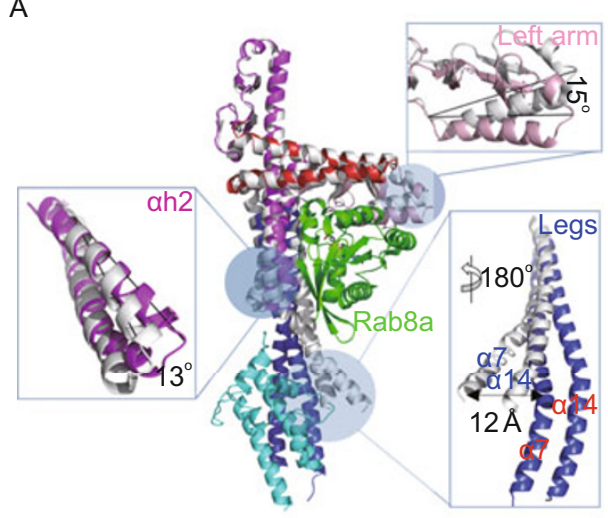

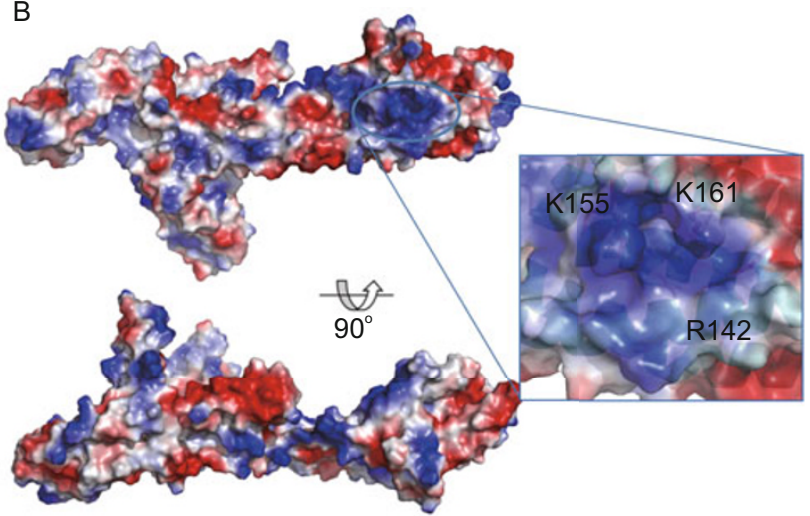

C Rab-free

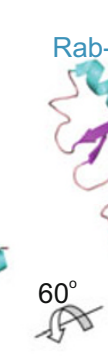

Cytoplasm

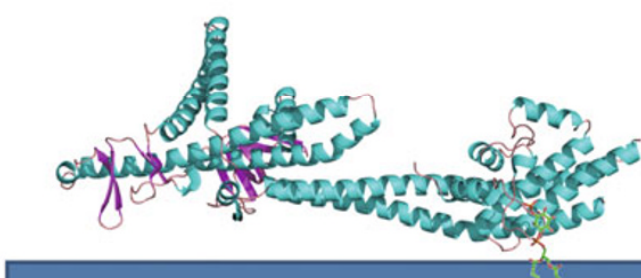

inding

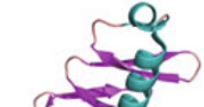

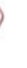

LCV membrane

\begin{abstract}
Figure 2. A possible working model for LidA orienting in the cytoplasm of host cell. (A) Structural superposition of LidA in apo-state and in complex with Rab8a. LidA ${ }_{60-594}$ is colored as in Fig. 1. LidA $201-583$ in the LidA-Rab8a complex is colored light gray and Rab8a is colored green. Three sites with remarkable conformational difference are highlighted and zoomed in to show details. (B) The electrostatic surface potential of $\mathrm{LidA}_{60-594}$ (calculated with PyMOL, positive in blue and negative in red). The speculated PtdlnsP binding pocket is surrounded by residues R142, K161 and K155. (C) A model for LidA orienting in the cytoplasm of host cell. LidA is shown with cartoon format and colored by secondary structure. LidA steps onto the cytoplasmic surface of LCV membrane by the binding of its right foot region to Ptdlns(3)P (green). Based on the rotation of the coiled-coil legs, LidA gets up to facilitate the Rab binding surface to interact with the membrane anchored Rab8a (green). The prenylated C-termini of Rab8a is colored orange.
\end{abstract}

of NEKGXXVXSLXDAH, where NEKG forms the first turn of the "square-knot" and LXD forms the conserved one-turn $3_{10}$ helix. In addition, all three canonical $\beta$-hairpins in two TBR motifs are also quite similar to each other, both structurally (with a r.m.s.d. of $0.87 \AA$, see Fig. 1G) and sequentially (Fig. S1).

The exact biological function of TBR motif remains unknown. Although the TBR of "left arm" is involved in Rab-binding of LidA, the TBR of "head" remains intact during Rab-binding. There is a possibility that TBR motif is also involved in the recognition and binding between LidA and partners other than Rabs. Further studies on the TBR motifs in LidA or identifications of new examples of TBR motif is required to obtain a deeper understanding.
Following the recently published structure of the LidA ${ }_{201-583}$ in complex with Rab8a (PDB entry: 3TNF) (Schoebel et al., 2011), the complex structures of the LidA $224-559$ with Rab1 (PDB entry: $3 T K L)$ or its mutant (PDB entry: 3SFV) (Cheng et al., 2012) was also reported. Both complex structures show a similar Rab binding mechanism of LidA and reveal the accurate binding interface of LidA to Rabs, majorly comprising a-helical hairpins ah1 and ah2, and the C-terminal a-helix of the left-arm TBR motif. Besides binding to Rab1 and Rab8, LidA is previously proved to associate with Rab6 (White et al., 1999; Machner and Isberg, 2006), a small GTPase regulating Golgi-to-ER membrane traffic, and more other Rab members were proved to associate with LidA in the newest work (Cheng et al., 2012). Considering that Rabs are highly conserved, their interaction with the Rabbinding domain of LidA may adopt the similar approach.

In comparison to the Rab-LidA complex structure, the LidA structure at an apo-state shows significant conformational changes. Structural alignment between LidA ${ }_{60-594}$ and Rab8a-LidA ${ }_{201-583}$ complex (PDB entry: 3TNF) indicates a roughly $60^{\circ}$ twist of the Rab-binding domain of the LidA (Fig. $2 \mathrm{C}$ ), resulting in a $12 \AA$ shift of the "foot-end" of the coiledcoil region ( $\alpha 7$ and $\alpha 14$ ) (Fig. 2A). In addition, two other regions providing the key interfaces with Rabs are also affected with certain allostery. The "left arm" of LidA $A_{60-594}$ moves about $15^{\circ}$ outward while the ah2 swings about $13^{\circ}$ outward 
in the Rab-free LidA structure compared to Rabs-complexed LidA. These observations suggest that the structure of LidA is more open in its free form and will contract slightly to hug abound Rab protein.

Recruitment of Rab protein to LCV by LidA is mediated by the membrane association of LidA through its PtdlnsP binding property, but the exact binding site is unclear. In our structure, the N-terminal right foot region (Pro60 to Ser181) of LidA forms a compact fold with a positively charged pocket (Fig. 2B) that is separated from the Rab-binding region by the $80-\AA$-long coiled-coil legs ( $\alpha 7$ and $\alpha 14)$. The pocket is surrounded by positively charged residues Arg142, Lys155 and Lys161 in a volume of $\sim 5 \times$ $5 \times 5 \AA$, which is comparable to the volume of the head of negatively charged Ptdlns(3)P or Ptdlns(4)P, suggesting this pocket may function in PtdlnsP binding. Similar charge features are observed in other Ptdlns(n)P binding proteins (Bravo et al., 2001). Thus, the right foot region may function in locating LidA to the cytosolic LCV membrane surface by binding to Ptdlns(3)P or Ptdlns(4)P and orient the Rab binding region to cytosol for recruiting Rabs.

To confirm and measure the affinity between LidA ${ }_{60-594}$ and Ptdlns(3)P, surface plasmon resonance (SPR) analysis was performed with Ptdlns(3)P and purified LidA $A_{60-594}$, because our attempts to obtain soluble expression of "right foot" alone failed entirely. The result revealed a relatively low affinity of Ptdlns(3)P and LidA $_{60-594}$, with a dissociation equilibrium constant $\left(K_{\mathrm{D}}\right)$ of approximately $3 \mathrm{mmol} / \mathrm{L}$ (Fig. S2). The weak interaction between Ptdlns(3)P and LidA $\mathrm{A}_{60-594}$ in vitro maybe in part due to that further conformational change of LidA is needed to enhance the binding affinity, or even other components are probably required to strength- en the interaction between them in vivo. However, the role of the N-terminal region in Ptdlns(n)P binding is necessary to be further measured experimentally in the future.

In summary, we propose a possible working model for LidA (Fig. 2C): After secreted out from the L. pneunophilavia Dot/lcm system, the human-body-like LidA steps on the cytoplasmic surface of LCV membrane by its right foot region and show its Rab-binding surface to cytosol for Rab recruitment. One of the two newly identified TBR motifs of LidA contributes to the binding of Rab proteins. Supported by the coiled-coil legs, the conformational changes induced by Rab-binding will make the central domain of LidA to re-orient to cytoplasm for Rab proteins to anchor on the membrane.

\section{FOOTNOTES}

This work was supported by grants from the National Basic Research Program (973 Program) (Nos. 2010CB911800 and 2011CB911101), the National Natural Science Foundation of China (Grant No. 30930020), and International Centre for Genetic Engineering and Biotechnology (ICGEB) (Project No. CRP/CHN09-01). Dr Shao Zhujun provided the genome of Legionella pneumophila.

Geng Meng, Xiaojing An, Sheng Ye, Yong Liu, Wenzhuang Zhu, Rongguang Zhang, and Xiaofeng Zheng declare that they have no conflict of interest.

This article does not contain any studies with human or animal subjects performed by any of the authors.

Geng Meng ${ }^{1,2^{*}}$, Xiaojing $\mathrm{An}^{3^{*}}$, Sheng $\mathrm{Ye}^{3}$, Yong Liu ${ }^{1,2}$, Wenzhuang Zhu ${ }^{1,2}$, Rongguang Zhang ${ }^{3 凶}$,

Xiaofeng Zheng ${ }^{1,2 \bowtie}$

${ }^{1}$ State Key Lab of Protein and Plant Gene Research, School of Life Sciences, Peking University, Beijing 100871, China
2 Department of Biochemistry and Molecular Biology, School of Life Sciences, Peking University, Beijing 100871, China

${ }^{3}$ National Laboratory of Biomacromolecules, Institute of Biophysics, Chinese Academy of Sciences, Beijing 100101, China

$\bowtie$ Correspondence: rzhang@ibp.ac.cn (R. Zhang), xiaofengz@pku.edu.cn (X. Zheng)

\section{REFERENCES}

Bravo, J., Karathanassis, D., Pacold, C.M., Pacold, M.E., Ellson, C.D., et al. (2001). Mol Cell 8, 829-839.

Brombacher, E., Urwyler, S., Ragaz, C., Weber, S.S., Kami, K., et al. (2009). J Biol Chem 284, 4846-4856.

Cheng, W., Yin, K., Lu, D., Li, B., Zhu, D., et al. (2012). PLoS Pathog 8, e1002528.

Conover, G.M., Derre, I., Vogel, J.P., and Isberg, R.R. (2003). Mol Microbiol 48, 305-321.

Derre, I., and Isberg, R.R. (2005). Infect Immun 73, 4370-4380.

Ensminger, A.W., and Isberg, R.R. (2009). Curr Opin Microbiol 12, 67-73.

Huber, L.A., Pimplikar, S., Parton, R.G., Virta, H., Zerial, M., et al. (1993). J Cell Biol 123, 35-45.

Machner, M.P., and Isberg, R.R. (2006). Dev Cell 11, 47-56.

Machner, M.P., and Isberg, R.R. (2007). Science 318, 974-977.

Muder, R.R., Yu, V.L., and Woo, A.H. (1986). Arch Intern Med 146, 1607-1612.

Roy, C.R., Berger, K.H., and Isberg, R.R. (1998). Mol Microbiol 28, 663-674.

Schoebel, S., Cichy, A.L., Goody, R.S., and Itzen, A. (2011). Proc Natl Acad Sci U S A 108, 17945-17950.

Tilney, L.G., Harb, O.S., Connelly, P.S., Robinson, C.G., and Roy, C.R. (2001). J Cell Sci 114, 4637-4650.

White, J., Johannes, L., Mallard, F., Girod, A., Grill, S., et al. (1999). J Cell Biol 147, 743-759.

Zhu, Y., Hu, L., Zhou, Y., Yao, Q., Liu, L., et al. (2010). Proc Natl Acad Sci U S A 107, 4699-4704.

* These authors contributed equally to the work. 